\title{
Raman threshold and optical gain bandwidth in silica fibers
}

\author{
G.S. Felinskyi ${ }^{1}$, P.A. Korotkov ${ }^{2}$ \\ Taras Shevchenko Kyiv National University, 2, Academician Glushkov prospect, 03022 Kyiv, Ukraine, \\ Phone: +38-044-526-0570, fax: +38-044-526-1073, \\ E-mail:'felinskyi@yahoo.com,2pak@mail.univ.kiev.ua
}

\begin{abstract}
Nonlinearity of the stimulated Raman scattering (SRS) process in single-mode fibers is the creation basis of the new class of modern photonic devices such as fiber Raman lasers and fiber Raman amplifiers. The quantitative analysis of the gain start conditions and the Raman laser threshold in the single mode fibers from the viewpoint of the strong gain description of active laser materials have been made in this work. It has been shown that the absolute transparency regime for optical transmission in fibers and Raman laser threshold for a monochrome signal wave can be directly obtained from the standard coupled equations using only fundamental fiber parameters. Limiting condition when material of fiber core starts transformation from the natural state with attenuation of the Stokes wave to the state in which the Stokes wave is amplified due to the pumping power may be expressed in a simple analytical form. The numerical data of laser threshold as a function of the wavelength and the examples of gain bandwidth determination for several widespread Raman fibers have been presented.
\end{abstract}

Keywords: optical fiber communication, fiber Raman laser, fiber Raman amplifier.

Manuscript received 23.09.08; accepted for publication 20.10.08; published online 11.11.08.

\section{Introduction}

The light amplification arising at stimulated Raman scattering (SRS) is widely applied to development of such photonic devices as fiber Raman lasers (FRL) [1] and fiber Raman amplifiers (FRA) [2]. SRS is nonlinear optical process and amplification of optical radiation results from coherent accumulation of Stokes wave intensity and it has strongly pronounced threshold character. The Raman gain threshold is defined doubly: first, as equality between the generated power of Stokes noise and pumping power at the fiber output [3] or second, as a laser threshold for the monochromatic wave, when fiber losses are fully compensated due to the Raman gain in single-mode fiber [4]. We will use the laser threshold definition for the analysis and quantitative calculations of occurrence conditions for the full transparency regime for optical signal transmission and the generation mode of coherent light in several widely used single-mode fibers in this work.

\section{Modeling basis}

Initial growth of the Stokes wave is defined by the pumping power, and it is described by the Raman gain coefficient $g_{R}$. The interaction between pumping and Stokes waves should be considered for calculation of the
Raman gain threshold. In the CW case, interaction of these propagated waves in the same direction is described by the following system of two coupled equations:

$$
\begin{aligned}
& \frac{d I_{s}(z, \omega)}{d z}=\mathrm{g}_{\mathrm{R}}(\omega) I_{p}(z) I_{s}(z, \omega)-\alpha_{s}(z) I_{s}(z, \omega), \\
& \frac{d I_{p}(z)}{d z}=\frac{\omega_{p}}{\omega_{s}} \mathrm{~g}_{\mathrm{R}}(\omega) I_{p}(z) I_{s}(z, \omega)-\alpha_{p}(z) I_{p}(z, \omega),
\end{aligned}
$$

where the absorption coefficients $\alpha_{s}$ and $\alpha_{p}$ account for the fiber losses at the Stokes and pumping frequencies, accordingly, $I_{s}$ is the Stokes intensity, $I_{p}$ is the pumping intensity. It should be noted that within the transparency windows of fiber $\alpha_{s} \approx \alpha_{p}=\alpha$, and attenuations $\alpha$ practically do not change within the whole band of Stokes shifted components of $\sim 1000 \mathrm{~cm}^{-1}$. For the transparency window near to $1.55 \mu \mathrm{m}$, this band corresponds to the range of wavelengths approximately $100 \mathrm{~nm}$, where attenuation $\alpha$ does not depend on the frequency and has a constant value in the diapason from 0.2 up to $1.0 \mathrm{~dB} / \mathrm{km}$, depending on the type of silica fiber.

Besides, it is considered that in Eqs. (1) and (2) the pumping is provided with a monochromatic wave possessing so narrow generated line that its spectral width can be neglected. Alternatively, the Stokes wave amplification $g_{\mathrm{R}}(\omega)$ takes place in a sufficiently wide 
bandwidth of optical frequencies. Accordingly, the Stokes wave intensity is the function not only of the variable $z$, but also $\omega$ as a parameter. The Raman gain coefficient is coupled to the cross-section of spontaneous Raman scattering which is experimentally measured size. It is possible to directly check up that the laser threshold is not only naturally determined using the standard coupled equations (1) and (2) for the SRS process, but also its frequency dependence can be obtained in the quantitative form being based only on fundamental material parameters of the fiber, that is, $g_{\mathrm{R}}(\omega)$ and $\alpha(\omega)$

Really, the differential equation (1) can have a special point in its right part, if the pumping power will change within the range wide enough. Therefore three cases are possible:

(i) if $d I_{s} / d z<0$, then there is attenuation of the Stokes wave;

(ii) if $d I_{s} / d z>0$, then there is amplification of the Stokes wave;

(iii) if $d I_{s} / d z=0$, then there is a special point.

The case (i) $d I_{s} / d z<0$ takes place when pumping is absent or at the low pumping power, and it corresponds to attenuation of a Stokes wave during distribution caused by its own losses in the fiber. Raman amplification (ii) of the Stokes wave is described by the product $g_{\mathrm{R}} I_{p}$, and it arises with growth of the pumping intensity $I_{p}$, when its absolute value starts to exceed the own losses $\alpha$. In this case, the fiber is transformed to the active gain media as the condition $d I_{S} / d z>0$ corresponds to some increase of the running Stokes wave in the propagation process along the fiber. In the intermediate case (iii) $d I_{s} / d z=0$, there comes an absolute transparency regime for Stokes wave propagation in the fiber as the Stokes intensity remains constant along fiber length because of full compensation of fiber losses.

The physical sense of the absolute transparency condition for a fiber closely corresponds to a laser threshold definition in the SRS process. As the pumping power $P_{p}(\omega)$ can be expressed using the pumping intensity $I_{p}(\omega)$ and the fiber effective area $A_{\text {eff }}$, the equation $d I_{s} / d z=0$ together with the equation (1) results in the following quantitative form:

$P_{p}^{\text {th }}=\frac{\alpha_{s} A_{\text {eff }}}{\mathrm{g}_{\mathrm{R}}(\omega)}$

for the limiting condition when the fiber core material starts its transformation from a natural state with dumping the Stokes wave to the state with Stokes wave amplification, when applying the pumping power. In contrast to the experimental threshold [5], the laser threshold (3) is defined using only material parameters of the fiber, and it does not depend on experimental arrangement, in particular, it does not depend on the fiber length. Often, as the $P_{p}^{\text {th }}$ value is chosen, its minimum takes place at $g_{\mathrm{R}}\left(\omega_{\max }\right)=g_{\mathrm{R} \max }$. Convenience of the given definition of the gain threshold will consist in the following. We shall assume that the pumping power value $P_{p}$ is known to us in some point $z$ of the fiber as a result of measurements or calculation. Then, using the known constant $\alpha$ and the determined function $g_{\mathrm{R}}(\omega)$ one can directly check the inequality $P_{p}>(<) P_{p}^{\mathrm{th}}$. If $P_{p}>P_{p}^{\text {th }}$, then not only set of frequencies satisfying the full transparency condition of the fiber is defined, but also the amplification (generation) band located between the pair of adjacent frequencies can be easily calculated. Specific examples of this modeling for the several widespread fibers are presented in the following section.

Thus, it is possible to directly calculate the Raman laser threshold as a function of frequency (or wavelength) in the Stokes shift range for any wavelength of a pumping source using (3), if the Raman gain profile $g_{\mathrm{R}}(\omega)$ is perfectly obtained. Sometimes, the gain profile $g_{\mathrm{R}}(\omega)$ is described using the simplified Lorentz lineform approximation. However, real fibers have so complex structure of amplification that satisfactory performance of $g_{\mathrm{R}}(\omega)$ function needs the special modeling. The exact enough model of multimode decomposition of the Raman spectrum on Gaussian type components has been developed by us recently [7].

Our model analysis of the Raman spectrum with Gaussian profiles is based on the following expression

$g_{\mathrm{R}}(\omega) \sim \sum_{i=1}^{N_{m}} A_{i} \exp \left[-\frac{\left(\omega-\omega_{v, i}\right)^{2}}{\Gamma_{i}^{2}}\right]$,

where $N_{m}$ is the number of modes used for decomposition and can be chosen in accord with the spectral range (typically, $N_{m}$ lies within the range of 10 to 13), $\omega_{v, i}$ is the central frequency of the $i$ th Gaussian profile, parameter $\Gamma_{i}=\mathrm{FWHM}_{i} /(2 \sqrt{\ln 2}) \approx 0.6 \cdot \mathrm{FWHM}_{i}$, where $\mathrm{FWHM}_{i}$ - full width at half maximum parameter of the $i$ th Gaussian profile that is usually used in spectroscopy. Amplitudes $A_{i}$ together with $\omega_{v, i}$ and $\Gamma_{i}$ we use as parameters for a nonlinear fitting procedure.

The result of Raman gain profile approximation is the individual set of $3 N_{m}$ numerical parameters for each fiber [7], which gives the performance $g_{R}(\omega)$ as the sum of exponents in (4). Exact approximation allows to essentially simplify the quantitative determination procedure of the pumping power that causes the full transparency regime for wave propagation in a specific fiber type.

\section{Modeling results and discussion}

Four types of silica fibers widely applied in optical communication for creation FRA and FRL were used for modeling [8]. Experimental gain profiles $g_{\mathrm{R}}(\omega)$ and the maximal values of the Raman gain coefficients $g_{\mathrm{R} \max }$ were perfectly measured earlier and taken from [6]:

$g_{\mathrm{R} \max }=0.4(\mathrm{~W} \cdot \mathrm{km})^{-1}$ for standard silica fiber, $g_{\mathrm{R} \max }=0.71(\mathrm{~W} \cdot \mathrm{km})^{-1}$ for TrueWave $\mathrm{RS}^{\mathrm{TM}}$ fiber type, 
$g_{\mathrm{R} \max }=3.1(\mathrm{~W} \cdot \mathrm{km})^{-1}$ for dispersion compensated fiber (DCF), and

$g_{\mathrm{R} \max }=6.3(\mathrm{~W} \cdot \mathrm{km})^{-1}$ for the special Raman fiber.

First three fiber types have received wide application in long distant communication links for optical signal transmission, and the fourth fiber type is mainly used in FRL. Pumping powers for Raman threshold causes the full fiber transparency as a function of the wavelength are shown in Fig. 1 for three types of communication fibers.

We used the pumping source with $\lambda_{p}=1.45 \mu \mathrm{m}$ supposed for definite calculation. Then the full transparency mode in the pure silica fiber (Fig. 1a) starts at the wavelength of $1.55 \mu \mathrm{m}$ when the pumping power achieves the value equal to $20.7 \mathrm{dBm}$ (117.5 mW). These values can be simply obtained graphically as follows. The horizontal line (marker) in Fig. 1 corresponds to the certain pumping power level of $P_{p}$, the latter moves from below upwards and displays how the pumping power grows. In the case of standard silica fiber (Fig. 1a) when the pumping power reaches $117.5 \mathrm{~mW}$, the marker line has one common point (touch) with the full transparency curve for this fiber. The cross point with abscissa corresponds to the wavelength $1.55 \mu \mathrm{m}$. If the pumping power grows, then the marker line will move upwards. Next, it is formed at least two crossing points of marker with the full transparency curve. The cross point with abscissa for this pumping power level corresponds to the wavelength of the full transparency when both absorption and amplification of a Stokes wave are equal to zero in accuracy for a studied fiber. The interval between these wavelengths of full transparency actually corresponds to the amplification bandwidth, as for every wavelength from this interval Raman gain exceeds own losses. Alternatively, outside the specified interval the Raman gain appears to be insufficient for full compensation of own losses, and signals with these frequencies will decay when propagating in the fiber.

In particular, the amplification band is equal to 6.7 THz (from 1513 up to $1566 \mathrm{~nm}$ ) in a standard silica fiber at the pumping power of $200 \mathrm{~mW}$ and the fiber losses $\alpha=0.2 \mathrm{~dB} / \mathrm{km}$ (Fig. 1a). The definition influence of the own losses on the Raman gain band of fibers is also enough simple. If the standard silica fiber will have losses of $0.3 \mathrm{~dB} / \mathrm{km}$, then the gain band for this fiber is essentially narrowed almost by 2 times up to $3.8 \mathrm{THz}$ (from 1533 up to $1563 \mathrm{~nm}$ ). The curve of the full transparency for this fiber is shown by a dotted line in Fig. 1a.

The amplification bands for both TrueWave $\mathrm{RS}^{\mathrm{TM}}$ fiber and DCF type used for communication are determined by us similarly between crossing points of the full transparency curve with the marker level of the pumping power, and results are shown in Figs 1b-c, accordingly. The pumping power was considered equal to $200 \mathrm{~mW}$ at all calculations for comparison.

The own losses $\alpha$ normally do not exceed $0.2 \mathrm{~dB} / \mathrm{km}$ for TrueWave $\mathrm{RS}^{\mathrm{TM}}$ fiber type. The level of $0.3 \mathrm{~dB} / \mathrm{km}$ shown in Fig. 1b can be considered as the top limit allowing to take into account additional losses in a cable (microbends, pressure, etc.) laid in real field conditions. In this case, the gain band of $10.2 \mathrm{THz}$ for $\alpha=0.2 \mathrm{~dB} / \mathrm{km}$ is reduced down to $7.3 \mathrm{THz}$, but remains essentially more wide as compared to the amplification band in the pure silica fiber at the same pumping power.

The special fiber to dispersion compensation (DCF) is most often applied for simultaneous Raman amplification of signals, as it has the greatest coefficient

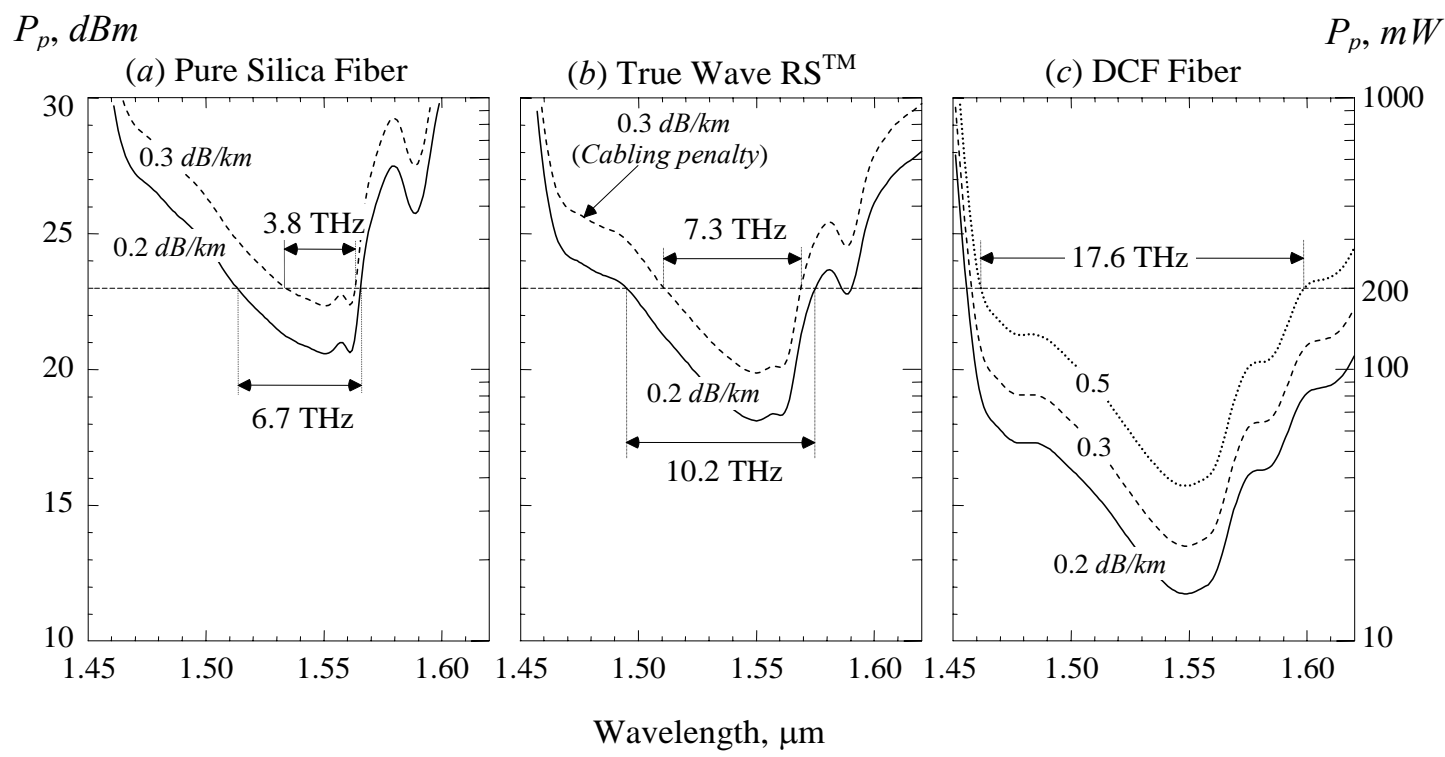

Fig. 1. Raman gain threshold $\left(\lambda_{p}=1.45 \mathrm{~nm}\right)$ as a function of the wavelength in single-mode fibers: $(a)$ - pure silica fiber; $(b)$ True Wave RS ${ }^{\mathrm{TM}}$; $(c)$ - dispersion compensated fiber (DCF). Every curve is the set of points of fiber full transparency. Gain bandwidth is shown for all fiber types at the probe pumping power of $200 \mathrm{~mW}$. 
$g_{\mathrm{R} \max }$ among the considered communication fibers. As a result, the amplification band in this fiber exceeds $17 \mathrm{THz}$ even if the fiber losses are equal to $0.5 \mathrm{~dB} / \mathrm{km}$ (see Fig. 1c). It should be noted that is its value almost 2 times larger than the total bandwidth of $\mathrm{C}+\mathrm{L}$ telecommunication windows. Therefore, it is possible to use considerably smaller pumping powers for effective Raman amplification in this fiber, because of the full transparency in this fiber is possible to start since $20 \mathrm{~mW}$ of the pumping power.

In contrast to the considered communication fibers, the special Raman fiber has $g_{\mathrm{R} \text { max }}=6.3(\mathrm{~W} \cdot \mathrm{km})^{-1}$, but it is mainly used for FRL fabrication. The matter is that the essential increase in Raman amplification of this fiber is achieved by an increased doping concentration of fiber core with $\mathrm{GeO}_{2}$ molecules (usually up to $20 \%$ ) during manufacturing this specialized Raman fiber. Unfortunately, this process is accompanied by appreciable increase of the own losses of the fiber that do not manage to be lowered less than down to the values $\alpha$ $0.3-1.0 \mathrm{~dB} / \mathrm{km}$. Thus, the Raman laser thresholds $\left(\lambda_{p}=1.45 \mu \mathrm{m}\right)$ as a function of the wavelength for this special Raman fiber are submitted in Fig. 2 with various values of their own losses that change within the range from 0.2 up to $1.0 \mathrm{~dB} / \mathrm{km}$. If the pumping power lies below the level of the laser threshold, which is located in the area under the curve in Fig. 2, Stokes waves possess attenuation, and Raman amplification takes place in the area above curves.

Naturally, the threshold power for a monochromatic Stokes signal at $\lambda_{s}=1.55 \mu \mathrm{m}$ will increase approximately from 9 up to $40 \mathrm{~mW}$ when the own fiber losses are increased from 0.2 up to $1.0 \mathrm{~dB} / \mathrm{km}$. Nevertheless, it is obvious from Fig. 2 that for rather small powers of pumping $\left(P_{p}=100 \mathrm{~mW}\right)$ in the Raman fiber with losses $\alpha=0.5 \mathrm{~dB} / \mathrm{km}$, the laser generation threshold begins at the wavelength of $1466 \mathrm{~nm}$ and is ended at the wavelength of $1613 \mathrm{~nm}$. The FRL retuning band potentially exceeds $140 \mathrm{~nm}$ in this case and covers both telecommunication windows. It is possible to expand the

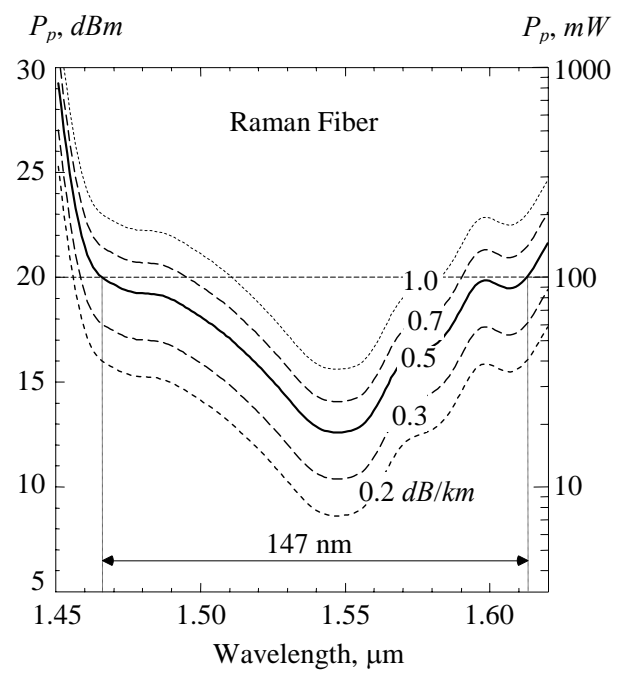

Fig. 2. Lasing threshold in a Raman fiber. retuning bandwidth of the laser even more, when the pumping power is increased or the own losses of the Raman fiber are decreased. The central wavelength inside this band of optical frequencies may be changed only by variation of the pumping wavelength, but not changing the active Raman fiber parameters.

\section{Conclusion}

The calculation technique to determine absolute values of the threshold pumping power that causes the full transparency conditions and the Raman amplification band in an arbitrary fiber were developed in this work for the set wavelength and power of a pumping source. The method is based only on frequency dependence of key fiber parameters such as Raman gain coefficient $g_{\mathrm{R}}(\omega)$ and the fiber attenuation parameter $\alpha(\omega)$. Quantitative ratings for the full optical transparency conditions at Raman amplification are presented for silica fibers widely used in optical communication. Modeling results on Raman gain bands above $10 \mathrm{THz}$ and nominal pumping powers are given for these fibers. It is shown that the laser threshold for a monochromatic Stokes signal will be observed irrespective of real fiber losses in a sufficiently wide spectral range, if power of pumping is raised up to several hundred milliwatts. In this case, laser generation can be received in the range approximately from 1.5 up to $1.6 \mu \mathrm{m}$ with the pumping wavelength $\lambda_{p}=1.45 \mu \mathrm{m}$ that corresponds to a full width of the maximal transparency window in silica fibers.

\section{References}

1. E.M. Dianov, A.M. Prokhorov, Medium-power CW Raman fiber lasers // IEEE J. Sel. Top. Quantum Electron. 6(6), p. 1022-1028 (2000).

2. M.N. Islam, Raman amplifiers for telecommunications // IEEE J. Sel. Top. Quantum. Electron. 8(3), p. 548-559 (2002).

3. R.H. Stolen, C. Lee, and R.K. Jain, Development of the stimulated Raman spectrum in single-mode silica fibres // J. Opt. Soc. Amer. B 1, p. 652 (1984).

4. P.A. Korotkov, G.S. Felinskyi, Fiber Raman CW lasers // Rev. Ukr. J. Phys. 3(2), p. 126-150 (2006).

5. G.P. Agrawal, Nonlinear Fiber Optics, Second ed. Academic., San Diego, CA, 1995.

6. K. Rottwitt, J. Bromage, A.J. Stentz, L. Leng, M.E. Lines, and H. Smith, Scaling of the Raman gain coefficient: applications to germanosilicate fibres // J. Lightwave Techn. 21(7), p. 1652-1662 (2003).

7. G.S. Felinskyi, Spectroscopic multiple-vibrationalmodelling of Raman gain for FRA design // Proc. SPIE/Ukraine 6(1-6), p. 418-426 (2006).

8. G.S. Felinskyi, P.A. Korotkov, Full transparency regime for optical transmission and lasing threshold in silica fibers due to nonlinear Raman interaction // Proc. $9^{\text {th }}$ Intern. Conf. on Laser and Fiber-Optical Networks Modeling (LFNM 2008), Oct. 2-4, Alushta, Crimea, Ukraine, p. 79-81 (2008). 